\title{
Optimization research on hinge points of the grader's working device based on ADAMS
}

\author{
Gao le ${ }^{1, \mathrm{a}}$, Hou Penglong ${ }^{2, \mathrm{~b}}$, Duan Junjie $\mathrm{e}^{3, \mathrm{c}}$ \\ ${ }^{1}$ Jiangsu XCMG Construction Machinery Research Institute Ltd Xuzhou, China \\ ${ }^{2}$ Jiangsu XCMG Construction Machinery Research Institute Ltd Xuzhou, China \\ ${ }^{3}$ Xuzhou XuGong Road Construction Machinery Ltd Xuzhou, China
}

\begin{abstract}
In order to study the hinge points load for grader's working device, a multi-body dynamic model of the grader is established for analyzing the construction condition and obtain the load of each hinge point. The analysis method is verified by prototype test. Taking the force of the key cylinder as the optimization objective, the positions of all hinge points are optimized. The result show that the scheme reduces the maximum force of the key cylinder by $26.5 \%$, which improve the stability of the grader.
\end{abstract}

\section{INTRODUCTION}

The grader's extensive auxiliary operating capability is due to its ability to perform 6 degrees of freedom in space[1-2]. The tooling device of grader is the main bearing structure, which is the key structure to complete these actions. The cylinder is the power to ensure the movement of the working device, whose stability directly relate to the stability of the vehicle. In particular, when digging ditches and scraping slopes, the blade can reach 90 degrees on the side, at which point the forces on the lifting cylinder and the centreshift cylinder reach the maximum, posing a challenge to the stability of the lifting cylinder and the centreshift cylinder. In recent years, the performance of the grader has been improved, the load of shoveling has been increased, and the potential instability of the lifting cylinder and the cable has been increased gradually, which affect the stability of the vehicle. Taking the grader as the research object, multi-body dynamics model of the vehicle was established on ADAMS which can be used in calculate the force on each hinge point of the working device under full working condition. In order to get the forces of the lifting cylinder and the centreshift cylinder, an actual vehicle test is carried out and the result is compared with the simulation.

In order to reduce the maximum load of the cylinder and improve the stability of the whole machine, the ground mechanic loading device hinge point location parameterized model is established, which takes enhancing the maximum load of suspension cylinder as the optimization goal. In the condition of shovel knife made slope angle, the optimization design of grader work device hinge point is carried out. After optimization, the forces on the left and right lifting cylinders and the centreshift cylinder are reduced, and the hinge points are arranged more rationally to achieve the optimization purpose.

\section{SIMULATION ANALYSIS OF GROUND MANEUVER MECHANICS}

\subsection{Establishment of dynamic simulation model}

According to the relative motion relation among the parts of the crane, corresponding motion pair is established in ADAMS[3-5], is shown in figure 1.The working device of the grader mainly work in three typical working modes, namely, shoveling, ditching and scraping. According to the different forms of the left and right lifting cylinders, the centreshift cylinder, the tip cylinders and the blade cylinders in the actual operation, the driving actions of the five cylinders in the multi-body dynamic model are designed with a total length of 300 steps. The driving actions of the left lifting cylinder is shown below:

Step(time, $41,0,55,-$

933)+step(time, 56,0,70,933)+step(time, 71,0,85,

322)+step(time, $86,0,100$,-

322)+step(time, 141,0,155,-

933)+step(time, 156,0,170,933)+step(time, 171,0

,185,322)+step(time, 186,0,200,-

$322)+$ step(time, $241,0,255$,-

933)+step(time, 256,0,270,933)+step(time, 271,0 ,285,322)+step (time, 286,0,300,-322).

The maximum traction force is applied at the shovel, and three driving forces are established considering the different loading positions of the shovel operation.

aleqing2009@163.com b1134957431@qq.com ckingdjj@126.com 
Driving function of the middle position of the blade: step(time,100,100000,101,0); Driving function of the right position of the shovel: step(time, 100,0,101, 100000)step( time,200,0,201,100000). The driver function for the left position of the blade: step(time,200,0,201,100000).

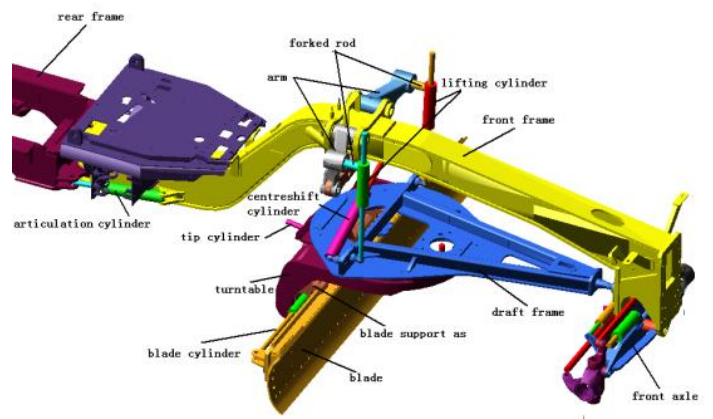

Fig. 1 Model diagram of main structure parts of grader

\subsection{Dynamic simulation calculation}

According to the settings above, a model is established in conditions of flat ground and small angle trench means whose arm and front frame pin hole in the middle. While the other model is established in conditions of large-angle trench working and the vertical slope working. The analysis results are shown in figure 2-3.

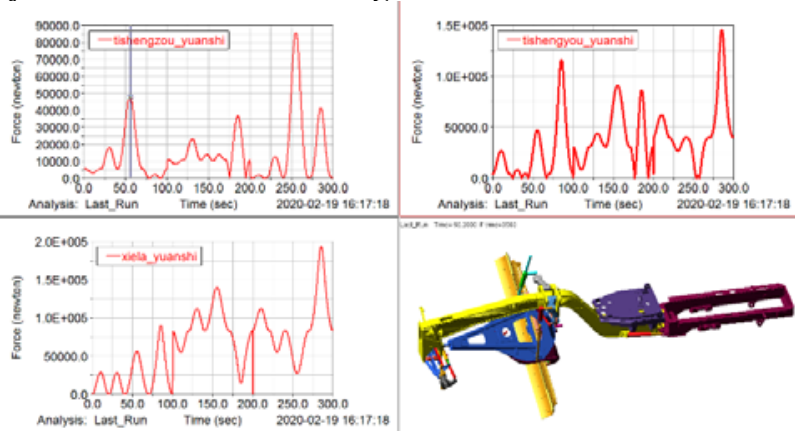

Fig. 2 Simulation results of ground working conditions and conditions of ditching and slope scraping at small angles

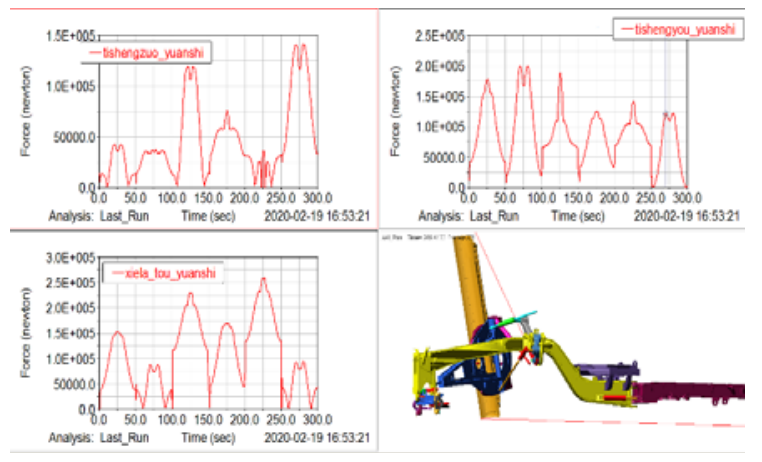

Fig. 3 Simulation results of large angle trench excavation and slope scraping

Based on the simulation, it can be found that the lifting cylinder force in the condition of flat ground is less than that in the condition of digging ditches and scraping slopes. The maximum force of the cylinder in the condition of ditching on the left side and vertical slope is greater than that in the condition of ditching and slope scraping on the right side. All the positions are shrinkage condition of the left lifting cylinder, extension condition of the right lifting cylinder, and loading of the blade at the left. The maximum force of the cylinder in the condition of ditching on the left side and vertical slope is greater than that in the condition of ditching and slope scraping on the right side.

\section{TEST AND COMPARISON WITH SIMULATION ANALYSIS}

\subsection{Test of hinge position}

In order to verify the correctness of the multi-body dynamic model, the test was carried out on the sample grader. Pressure sensors was installed on both sides of lifting cylinders. After fixing the other end of the pull rope and adjusting the attitude of the working device, to make the traction frame horizontal and symmetrical on the left and right sides and keeping the back of the blade vertical and symmetrical on both sides, what was showed in figure 4 , then the sample grader was Started the motor slowly by keeping the tire moving towards the head.

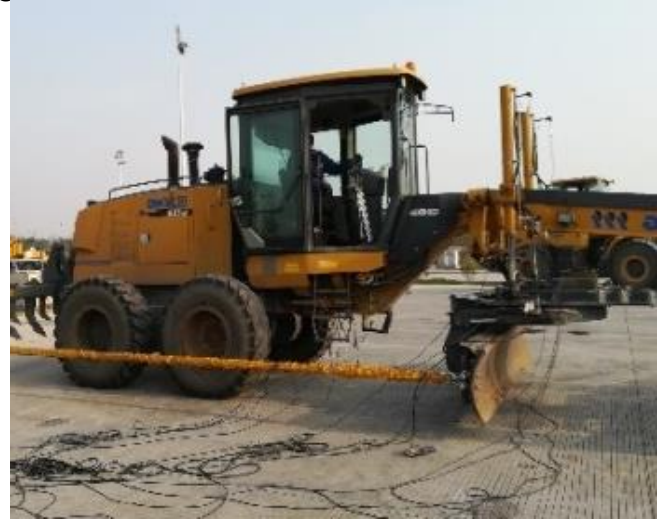

Fig. 4 Grader test site

\subsection{Comparison of test and simulation analysis}

According to the cylinder size calculation, the forces data were obtained. While the test data was obtained and showed in the Table 1. Then loading the data of the actual tension sensor to get the force of the lifting cylinder and the centreshift cylinder. After calculation, it was found that the maximum error between the measured cylinder force and the calculated cylinder force under the same attitude is within $7 \%$, which meets the engineering practical application.

Table 1 Comparison table of experimental and simulation analysis data

\begin{tabular}{|c|c|c|c|c|}
\hline & $\begin{array}{c}\text { Loading } \\
\text { force } \\
\mathrm{t}\end{array}$ & $\begin{array}{c}\text { Left } \\
\text { lifting } \\
\text { cylinder } \\
\mathrm{N}\end{array}$ & $\begin{array}{c}\text { Right } \\
\text { lifting } \\
\text { cylinder } \\
\mathrm{N}\end{array}$ & $\begin{array}{c}\text { Centreshift } \\
\text { cylinder } \\
\mathrm{N}\end{array}$ \\
\hline Test & 0 & -13589 & -13830 & -1225 \\
\hline
\end{tabular}




\begin{tabular}{|l|c|c|c|c|} 
data & 2.3 & -5367 & -10757 & -15974 \\
\cline { 2 - 5 } & 4.1 & 1727 & -7400 & -29284 \\
\cline { 2 - 5 } & 6 & 10176 & -3890 & -44440 \\
\cline { 2 - 5 } & 7 & 13141 & -2206 & -50870 \\
\hline \multirow{4}{*}{$\begin{array}{l}\text { Analysis } \\
\text { data }\end{array}$} & 0 & -13022 & -13138 & -1155 \\
\cline { 2 - 5 } & 2.3 & -5012 & -10223 & -16770 \\
\cline { 2 - 5 } & 4.1 & 1600 & -6960 & -30420 \\
\cline { 2 - 5 } & 6 & 9555 & -3929 & -46010 \\
\hline \multirow{4}{*}{$\begin{array}{l}\text { Percent } \\
\text { error } \%\end{array}$} & 7 & 12562 & -2248 & -52310 \\
\cline { 2 - 5 } & 2.3 & $-4 \%$ & $-5 \%$ & $-6 \%$ \\
\cline { 2 - 5 } & 4.1 & $-7 \%$ & $-5 \%$ & $5 \%$ \\
\cline { 2 - 5 } & 6 & $-6 \%$ & $1 \%$ & $4 \%$ \\
\cline { 2 - 5 } & 7 & $-4 \%$ & $2 \%$ & $3 \%$ \\
\hline
\end{tabular}

\section{OPTIMIZATION OF HINGE POSITION}

\subsection{Determination of objective function}

Through above analysis, it is found that the limit working condition of grader is the condition of the shovel at 90 degrees. Under this working condition, the load of cylinder force reaches the maximum, which increase danger to the stability of the cylinder, which is took as the optimization objective.

\subsection{Determination of design variables}

Establish optimization variables for all hinge points involved in the working device of the grader and correlate the relationships among variables[6-7], to ensure that there is only one optimization variable for the same parameter and make sure that the constraint pair can move with it.

Table 2 Optimization variable information table

\begin{tabular}{|c|c|c|c|}
\hline variable & $\mathrm{X}$ & $\mathrm{Y}$ & $\begin{array}{ll}Z \\
\end{array}$ \\
\hline ZXT_you & $($ ZXT_zuo.loc_x) & (ZXT_zuo.loc_y) & $\begin{array}{c}(-1 \\
* \text { *ZXT_zuo.loc_z } \\
\end{array}$ \\
\hline ZXT_zuo & (DV_1) & (DV_2) & (DV_3) \\
\hline ZXW zuo & (DV 4) & (ZXT zuo.loc y) & (DV 5) \\
\hline ZXW you & (ZXW zuo.loc $\mathrm{x})$ & (ZXT_zuo.loc y) & $\begin{array}{c}\left(-1{ }^{*}\right. \\
\text { ZXW zuo.loc z }) \\
\end{array}$ \\
\hline BJ_you_y & (BJ_zuo_z.loc_x) & (BJ_zuo_z.loc_y) & $\begin{array}{c}\left(-1^{*}\right. \\
\text { BJ_zuo_z.loc_z })\end{array}$ \\
\hline BJ zuo z & (DV_6) & (DV 7) & (DV 8) \\
\hline BJ_you_z & (BJ_zuo_y.loc_x) & (BJ_zuo $y \cdot$ loc $\left.\_y\right)$ & $\begin{array}{c}(-1 * \\
\text { BJ_zuo_y.loc_z })\end{array}$ \\
\hline BJ zuo y & (DV 9) & (DV 10) & (DV 11) \\
\hline liang you & (liang zuo.loc $\mathrm{x}$ ) & (liang zuo.loc y) & $\begin{array}{c}(-1 * \\
\text { liang } \\
\text { zuo.loc } \mathrm{z}) \\
\end{array}$ \\
\hline liang_zuo & (DV_12) & (DV_13) & (DV_14) \\
\hline liang zhong & (liang zuo.loc $x$ ) & (DV 15) & 0 \\
\hline xiela tou & (DV 16) & (DV 17) & (DV 18) \\
\hline xiela_wei & (DV 19) & (DV 20) & (DV 21) \\
\hline TS zuo $x$ & (DV 22) & (DV 23) & (DV 24) \\
\hline TS_you_ $\mathrm{x}$ & (TS_zuo_x.loc_x) & (TS_zuo_x.loc_y) & $\begin{array}{c}\left(-1^{*}\right. \\
\text { TS_zuo_x.loc_z) }\end{array}$ \\
\hline JWQ zuo & (DV 25) & (DV 26) & (DV 27) \\
\hline JWQ_you & (JWQ_zuo.loc_x) & (JWQ_zuo.loc_y) & $\begin{array}{c}\left(-1^{*}\right. \\
\text { JWQ Zzuo.loc_z }) \\
\end{array}$ \\
\hline JWG wei & (DV 28) & (DV 29) & 0 \\
\hline
\end{tabular}

\begin{tabular}{|l|l|l|l|}
\hline JWG tou & (DV 30) & (DV 31) & 0 \\
\hline
\end{tabular}

\subsection{Determination of constraint conditions}

The realization of blade 90 sideslip is an important performance index of grader operation. On this basis, the optimization of hinge point position is implemented. Therefore, the secondary constraint condition of multibody dynamic analysis is that the blade can achieve left and right 90 degree lateral.

\subsection{Sensitivity analysis}

The maximum force of the left lifting cylinder, the right lifting cylinder and the centreshift cylinder was taken as the optimization objective to obtain their sensitivity. The sensitivity optimization information of the key position was selected in table 3 .

Table 3 Sensitivity information table

\begin{tabular}{|c|c|c|c|c|c|c|}
\hline DV_6 & $\begin{array}{c}\text { Left } \\
\text { lifting } \\
\text { cylinder } \\
1 \mathrm{E}+04\end{array}$ & $\begin{array}{c}\text { Sensi- } \\
\text { tivity }\end{array}$ & $\begin{array}{c}\text { Right } \\
\text { lifting } \\
\text { Cylinder } \\
1 \mathrm{E}+05\end{array}$ & $\begin{array}{c}\text { Sensi- } \\
\text { tivity }\end{array}$ & $\begin{array}{c}\text { Centreshift } \\
\text { cylinder } \\
1 \mathrm{E}+05\end{array}$ & $\begin{array}{c}\text { Sensi- } \\
\text { tivity }\end{array}$ \\
\hline-2798.0 & 8.5830 & -5.4 & 1.5022 & -103.2 & 1.9535 & -46.3 \\
\hline-2781.3 & 8.5740 & -4.5 & 1.4850 & -99.2 & 1.9458 & -41.4 \\
\hline-2764.7 & 8.5680 & -2.7 & 1.4692 & -91.0 & 1.9397 & -39.6 \\
\hline-2748.0 & 8.5650 & -0.9 & 1.4547 & -87.9 & 1.9326 & -39.9 \\
\hline-2731.3 & 8.5651 & 0.9 & 1.4399 & -87.3 & 1.9264 & -34.2 \\
\hline-2714.7 & 8.5681 & 2.8 & 1.4256 & -84.5 & 1.9212 & -34.9 \\
\hline-2698.0 & 8.5743 & 3.7 & 1.4117 & -83.2 & 1.9148 & -38.4 \\
\hline
\end{tabular}

\section{OPTIMIZATION DESIGN AND ANALYSIS}

According to the sensitivity analysis results, the following two technical schemes are shown in figure 5. The optimized scheme is analyzed, and the analysis results are shown in figure 6-7.

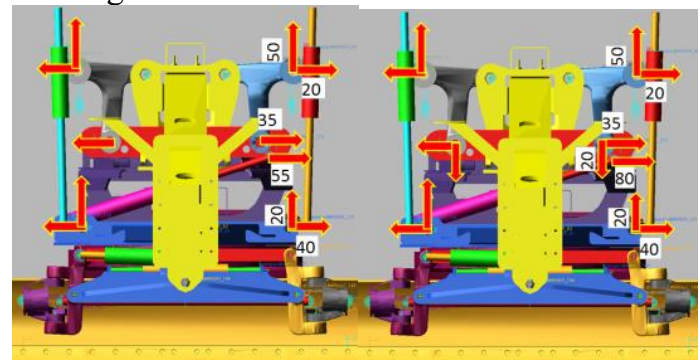

Fig. 5 Optimization scheme diagram of hinge point position

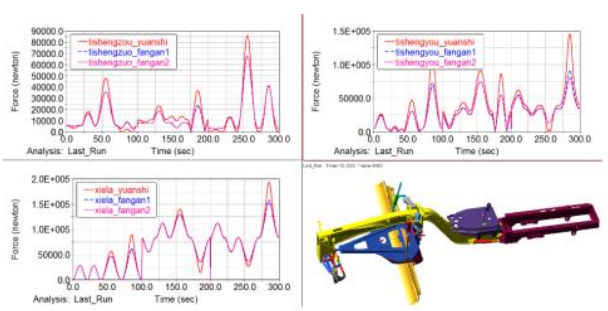

Fig. 6 Simulation comparison before and after optimization of ground working conditions, ditching and slope scraping at small angle 


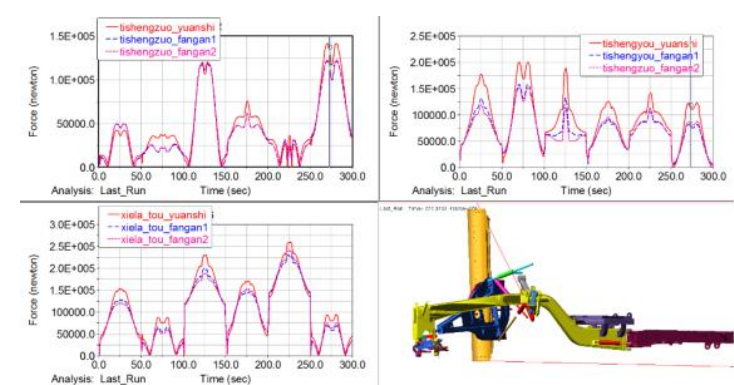

Fig. 7 Simulation comparison before and after optimization of large angle trench excavation and slope scraping

The simulation results after the optimization of ground working condition and small Angle trench excavation condition are as follows: in scheme 1, the maximum force on the middle left lifting cylinder was reduced by $21.1 \%$; the maximum force on the right lifting cylinder was reduced by $37.6 \%$. The maximum force of the centreshift cylinder was reduced by $18.3 \%$. In scheme 2 , the maximum force on the middle left lifting cylinder was reduced by $21.7 \%$; the maximum force on the right lifting cylinder was reduced by $45.1 \%$; the maximum force on the centreshift cylinder was reduced by $21.0 \%$.

The simulation results of the optimized conditions of large Angle trench excavation and scraping are as follows: In scheme 1: the maximum force on the middle left lifting cylinder was reduced by $13.3 \%$; the maximum force on the right lifting cylinder was reduced by $24.5 \%$; the maximum force on the centreshift cylinder .In scheme 2, the maximum force on the middle left lifting cylinder was reduced by $14.3 \%$; the maximum force on the right lifting cylinder was reduced by $26.5 \%$; the maximum force on the centreshift cylinder was reduced by $11.1 \%$.

The two schemes are optimized, and the maximum lateral angles of the right and left sides of the blade could reach 90 degrees. After comprehensive consideration, scheme 2 was selected.

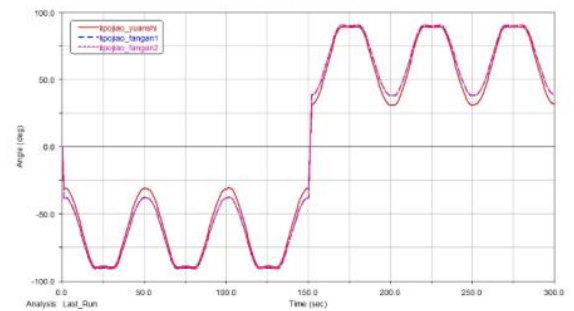

Fig. 8 Comparison of the maximum lateral angle of the shovel before and after optimization

\section{SUMMARY}

(1) In this paper, a hinge point position optimization method based on the vehicle model is formed, and its correctness is verified by test. The optimization scheme reduces the maximum force of the left lifting cylinder by $14.3 \%$, the maximum force of the right lifting cylinder was reduced by $26.5 \%$, and the maximum force of the centreshift cylinder was reduced by $11.1 \%$, and the parameters are applied to the product improvement design.

(2) Through multi-body dynamics, it is found that the 90-degree side-running condition is the ultimate operating condition of the grader, and the maximum cylinder force in the condition of ditching on the left side is greater than the one in the condition of ditching and slope scraping on the right side.

\section{REFERENCES}

1. Rusjin. Dynamic analysis and research on the rotating ring of working device of grader $[\mathrm{J}]$. Construction machinery technology and management. 2011 (8) : 110-112.

2. Lei WANG, Xin ZOU, Peng-Xiong LIN. Dynamics Simulation of 6-DOF Assemblying Manipulator Based on ADAMS. [J]Advances in Engineering Research (AER), volume 131(2017) 437 442

3. Chen Liping, Zhang yunqing, Ren weiqun et al. Dynamic analysis of mechanical systems and application of Adams [M]. Tsinghua university press, 2005.

4. Jia Changzhi, Yin junhui, Xue wenxing et al. ADAMS virtual prototype from entry-level to proficient [M]. Beijing: mechanical industry press, 2010.01

5. Chen Fenghua.ADAMS virtual prototyping technology from the beginning to master 2016[M]. Tsinghua university press, 2016.

6. Z. Zhang, L. Xu, Y. Y. Tay, P. Flores and H. Lankarani,Multiobjective optimization of mechanisms with clearances in revolute joints, In New Trends in Mechanism and Machine Science, Mechanisms and Machine Science 24, P. Flores And F. Viadero (Eds.), Springer International Publishing,Switzerland (2015).

7. M. Ahmedalbashir, L. Romdhane and J. Lee. Dynamics of a four-bar mechanism with clearance and springs Modeling and experimental analysis. [J] Journal of Mechanical Science and Technology 31 (3) (2017) 1023 1033 